\title{
Excitatory Action of Extracellular ATP on Chick Skeletal Muscle
}

\author{
STEVEN A. THOMAS AND RICHARD I. HUME ${ }^{a}$
}

\author{
Department of Biology \\ Natural Science Building \\ University of Michigan \\ Ann Arbor, Michigan 48109
}

Micromolar concentrations of extracellular ATP elicit a rapid excitatory response in developing chick skeletal muscle.' These experiments were designed to determine the ionic basis of the excitatory response. Experiments were performed on myoballs using the whole-cell patch-clamp technique. The reversal potential of the excitatory response was consistent with an increase in conductance to either cations or anions. To distinguish between these possibilities, myoballs were bathed with a solution that would produce a negative reversal potential for cations, and a positive reversal potential for anions. The ATP reversal potential was between the two extremes, indicating that both cations and anions were permeant. In contrast, acetylcholine responses always reversed close to the predicted cation reversal potential. We estimated the relative permeability of ions by measuring the shift in reversal potential when one ion was
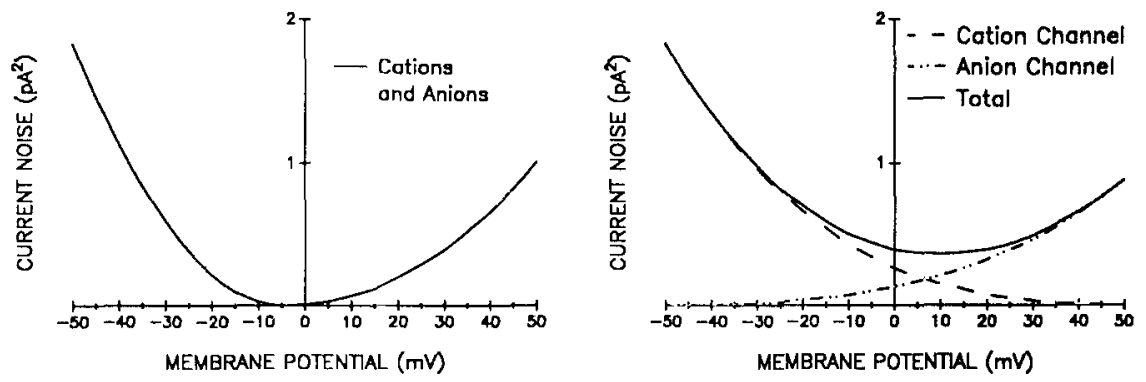

FIGURE 1. Theoretical basis for deciding whether ATP activates a single class of nonselective channels or separate cation and anion channels during the excitatory response. As first pointed out by Dionne and Ruff, if the reversal potentials of two types of permeant ions are set far apart, the predicted noise is very different for the case of one or two channels. For any single channel type, the noise is the product of a term characteristic of the behavior of the single channels times the driving force squared $\left(\operatorname{Var}_{1}=K\left(V_{\mathrm{m}}-E_{\mathrm{rcv}}\right)^{2}\right)$. If a single class of nonselective channels is involved, then the noise should go to zero at the macroscopic reversal potential (left panel). In contrast, if two different channel types are involved, then the noise at any potential will be the sum of the noise through the two sets of channels. Because neither type of channel is at its reversal potential at the macroscopic reversal potential, noise should not go to zero (right panel).

${ }^{a}$ To whom correspondence should be sent. 
TABLE 1. Measurements of ATP-Activated Noise in Myoballs

\begin{tabular}{lccc}
\hline & \multicolumn{3}{c}{ Potential (mV) } \\
\cline { 2 - 4 } & -50 & -5 & +40 \\
\hline Current (pA) & $-223 \pm 31$ & $-1 \pm 6$ & $+200 \pm 86$ \\
Variance (pA $\left.{ }^{2}\right)$ & $1.83 \pm 0.61$ & $0.00 \pm 0.04$ & $0.66 \pm 0.12$ \\
Numbers of cells & 10 & 11 & 4 \\
\hline
\end{tabular}

"The reversal potential for cations was set near $+50 \mathrm{mV}$, and the reversal potential for anions near $-50 \mathrm{mV}$, by making whole-cell recordings with appropriate solutions (external solution: $150 \mathrm{mM} \mathrm{CsNO}_{3}, 3 \mathrm{mM} \mathrm{TEA} . \mathrm{OH}, 1 \mathrm{mM} \mathrm{BaCl}, 10 \mathrm{mM}$ HEPES, and $40 \mathrm{mM}$ glucose; internal solution: $21 \mathrm{mM} \mathrm{CsNO}, 43 \mathrm{mM}$ TEA.OH, $10 \mathrm{mM}$ BAPTA, $1 \mathrm{mM} \mathrm{CaCl}, 10 \mathrm{mM}$ HEPES, and $200 \mathrm{mM}$ sucrose). With these solutions the macroscopic reversal potential of the excitatory ATP response was $-5 \mathrm{mV}$. Although there was a clear increase in ATP-activated noise at -50 and +40 , there was no excess noise at the reversal potential. We conclude that there is a single class of nonselective channels activated by ATP. Based on the ratio of the variance to the mean, we estimate that the unitary conductance of these channels is about $0.3 \mathrm{pS}$.

substituted for another. We found that small monovalent cations, small divalent cations, and small anions all permeate the membrane during the excitatory ATP response, and that there was only moderate selectivity between many of these ions. However, large organic cations and anions (such as tetraethylammonium and glucuronate) were not permeant.

To determine whether ATP activates a single class of channels that conduct both cations and anions, or whether ATP activates separate cation and anion channels, we analyzed the fluctuations about the mean current induced by ATP. As first pointed out by Dionne and Ruff, if the reversal potentials of two types of permeant ions are set far apart, the predicted noise is very different for the cases of one and two channels. For any single channel, the noise is the product of a term characteristic of the behavior of the single channels $(K)$ times the driving force $\left(V_{\mathrm{m}}-E_{\mathrm{rev}}\right)$ squared. If a single class of nonselective channels is activated by ATP, then the noise should go to zero at the macroscopic reversal potential (FIG. 1, left panel). In contrast, if two different classes of channels are involved, then the noise at all potentials will be the sum of the noise through the two sets of channels. Because neither channel is at its reversal potential at the macroscopic reversal potential, noise should not go to zero (FIG. 1, right panel). In our experiments ionic conditions were arranged so that the reversal potential for cations was $+50 \mathrm{mV}$ and the reversal potential for anions was $-50 \mathrm{mV}$. At both $+40 \mathrm{mV}$ and $-50 \mathrm{mV}$ ATP elicited a clear increase in noise, but at the ATP reversal potential of $-5 \mathrm{mV}$ no increase in noise above background was seen (TABLE 1). These results indicate that there is only a single excitatory ATP-activated channel type, which does not select by charge. Analysis of the current variance indicates that the unitary conductance of the channel is approximately $0.3 \mathrm{pS}$.

\section{REFERENCES}

1. Hume, R. I. \& M. G. Honig. 1986. Excitatory action of ATP on embryonic chick muscle. J. Neurosci. 6: 681-690.

2. Dionne, V. E. \& R. L. RUFF. 1977. Endplate current fluctuations reveal only one type of channel type at frog neuromuscular junction. Nature 266: 263-265. 